\title{
An Empirical Study of Memory and Information Retrieval with a Spatial User Interface
}

\author{
Chiladda Chennawasin, Janet Cole, Chaomei Chen \\ Department of Information Systems \& Computing \\ Brunel University \\ Uxbridge UB8 3PH, UK \\ Tel: +44 1895203080 \\ Fax: +44 1895251686 \\ Email: \{chiladda.chennawasin, janet.cole, chaomei.chen\}@brunel.ac.uk
}

\begin{abstract}
The rapid advances in information retrieval, especially with visualisation-oriented user interface design, highlight the need for an understanding of the interplay between cognitive abilities of users and the design of visual user interfaces. In this study, we focus on two aspects of memory abilities of users in attempts to reveal their roles in facilitating information-seeking tasks. In particular, we study the relationships between associative memory and the use of a spatial user interface, and the relationship between visual memory and the same user interface. The design of the spatial user interface draws upon information visualisation techniques in order to highlight the strongest semantic relations in the search space. Two retrieval tasks were performed. While associative memory was strongly correlated with the first task, visual memory was negatively correlated with the second task. Both correlations were statistically significant. Implications for user interface design and suggestions for further empirical studies are also discussed.
\end{abstract}

Keywords: Visual information retrieval, memory, information visualisation, empirical study

\section{Introduction}

It is difficult for the user to find information of interest within a large information space. A 3D representation of the user interface may be helpful in assisting information retrieval task. The problem in virtual environment design is the lack of a structural framework and directional cues to which the user can relate. Users consequently, may easily become disoriented and unable to search for destinations or construct an accurate cognitive map of the space. Inconsistency between abilities exhibited by individual users may be a factor that influences the design of a user interface which is to be investigated. As a result users of mixed ability are able to take advantage of the concept upon which the use interface is designed to greater benefit.

In this empirical study, therefore, we aim to determine the question: Do users, with higher memory capability, navigate more effectively in virtual environment? Do users, with higher ability to recall associate items, perform better task? Do users, with higher visual memory, find it's easier to use 3D user interface? If so, a good structural visualisation user interface is particularly useful to people with lower memory capability.

\section{Background}

There have been a number of works exploring navigation in virtual environments. Researchers have investigated whether human factors influence the design of the user interface for large information space $[3,4,5,6,9,10,12]$. Their studies demonstate that a well-designed user interface will accommodate individual differences.

For example, Swan and Allan [12] measured cognitive factors and information retrieval. They tested users verbal fluency and their spatial abitilty and found correlation worthy of further investigation.

Darken and Sibert [5,6], tested environment navigational cues within virtual worlds. They found that people tend to take advantage of environment cues which make it easier to locate search targets. Individuals showed different behaviour when they used different tools. Without landmarks, paths or cues, subjects are inclined to become disorient in virtual worlds.

The navigation work of Darken and Sibert links with that of Chun and Jiang [4] who conducted experiments into how visual context influences visual search and identification tasks. Their experiment tested location recall. A significant benefit was found to exist due to the contextual cueing effect, which was predictive of performance.

Additional navigational research was performed by Leplow, Höll, Zeng and Mehdorn [9], who conducted research into spatial orientation and spatial memory. They examined spatial abilities to detect problem solving behaviour, dissociation of place, landmark and response strategies. They assessed cue dependent orientation and showed that it is possible to identify different types of spatial problem-solving strategies. 
Whereas Mallot, Gillner, van Veen and Bülthoff [10], looked at three mechanisms of spatial memory: path integration, guidance and direction. They show that spatial relations can be learned from exploration in a virtual environment even one that may be subject to restricted viewing conditions. They have four important findings. The effect of viewing condition found that the error rate dropped as more information was provided. Transfer and latent learning showed that there was a reduced error rate for later search tasks compared with earlier ones. Persistence indicated that subject's movement decisions were based on simple associations, and subject differences being the individual differences of the test subjects.

Subject differences effecting performance results were also observed during the work of Chen and Czerwinski [3] who conducted an empirical study in an attempt to understand the role of individuals' spatial abilities in virtual environmental navigation. They found that the visualised semantic stucture of 3D user interface facilitated users' navigation strategies and their spatial memories. In addition, users' spatial memory were significantly correlated with their spatial abilities. We extend the work of Chen and Czerwinski whereas our research distinguishes the effect upon associative and visual memory of a spatial user interface. All these studies suggest that spatial user interface design is closely related to individual user cognitive ability.

\section{Human Memory}

Humans differ in how they handle the same information and confront the same problem. An explanation of the difference in cognitive style and strategies such as learning and recall is probably due to the memory abilities of each individual. In our study, we focus on two aspects of memory abilities, associative memory and visual memory, of users in attempts to reveal their roles in facilitating information-seeking tasks.

Associative memory involves the ability to recall one part of a previously learned when the other part of the pair is presented. Using the picture-number combination (see Fig. 1) tests this ability. The test consists of pictures and numbers. After studying both the pictures with the numbers, the subjects are required to memorise their association and reproduce them.

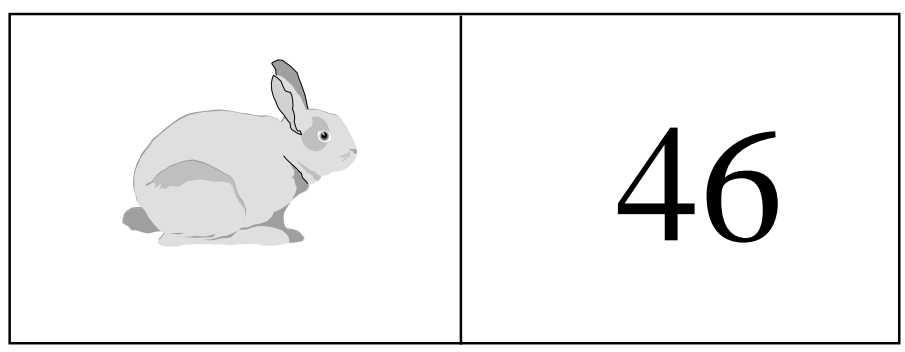

Figure 1: Picture-Number Combination

The other part of our study is visual memory, which is the ability to remember the configuration, location, and orientation of figural material. Using the shape memory test (see Fig. 2), the ability to remember a group of shapes and their positions in relation to each other is tested. Subjects are required to study the image, noting the different shapes and their orientation. They are then presented with similar grouped images and they must indicate which of them match in shape and orientation.

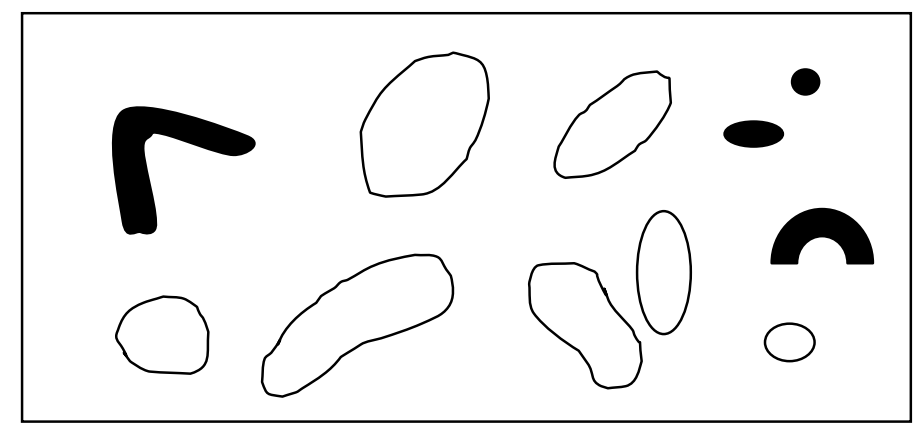

Figure 2: A Group of Shapes and Their Position in Relation to Each Other 


\section{Method}

The following method was used in the empirical study.

\subsection{Subjects}

Ten subjects, six males and four females raging in age from 25 to 40 participated. All subjects were computerliterate, but with varying degrees of experience with virtual environments.

\subsection{User Interface Design}

The user interface prototype was designed by Chen [1,2]. It is a hypermedia information system emphasising the role of intrinsic inter-document relationships in structuring and visualising a large hypermedia information space. Salient patterns in these relationships are extracted and visualised in a simple and intuitive associated network. The spatial layout of a visualisation is optimised such that closely related documents are placed near to each other and only those with an intrinsic connection among them are shown to users as automatically generated virtual links. Further details of models and algorithms can be found in [1,2].

The semantic space of the user interface consists of 169 papers published in the three ACM SIGCHI conference proceedings (ACM 1995, ACM 1996 and ACM 1997). According to semantic similarities, papers are structured and visualised as similar topics papers tended to be grouped near to each other. A virtual link structure was also automatically generated. Papers are visualised as coloured spheres in a connected network.

The user interface was based on the Netscape Comunication 4.0 browser and its COSMO Player 2.0 viewer. Splitting the screens into two frames, the virtual world was displayed in the left-hand side and the abstract of the papers selected was displayed in the other side (see Fig. 3). Colour spheres of each paper are labelled with the initials of authors. When the users point the mouse cursor at a sphere, the title of the paper pops up. Alternatively, if the users click on the sphere, the abstract will appear in the right-hand side frame. The users can operate the virtual world in various ways, for example, they can walk towards the world by dragging the mouse forward. By reversing this process, the users can overview the entire space at a glance.

The abstracts of the papers are stored on a web server at Brunel University. The experiment was conducted on a PC with $233 \mathrm{MHz}$ CPU and $32 \mathrm{Mb}$ RAM, with a 17-inch display monitor.

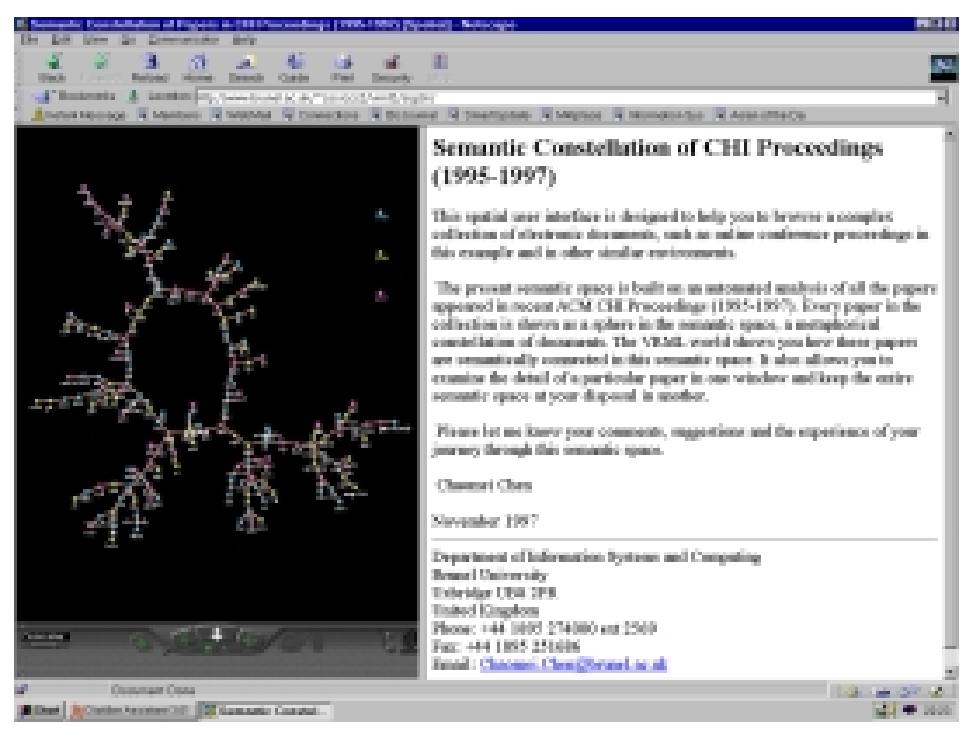

Figure 3: The User Interface of CHI Proceedings

\subsection{Procedure}

The study included pre-test and post-test phases. All subjects were arranged to perform the test, under a neutral mood, which was created through the medium of general conversation, in the following areas.

- Memory pre-tests. Associative memory and visual memory scores were collected from the standard memory test of [7]. Each test contained two parts and required apploximately fifteen minutes to complete the task. 
- $\quad$ Pre-test Usability questionnaires. The questionnaire was developed by Microsoft usability engineers. The questions ask about typical computer-use of the subjects.

- $\quad$ Tests. On the following day, subjects were asked to perform two tasks using the designed prototype. An explanation and a demonstation on how to use the COSMO Player 2.0 was given to the subjects. It was ensured that all subjects were familiar with the commands to manipulate the virtual word.

Each subject was given ten minutes for Task 1. In Task 1, subjects were asked to find as many papers as they could on the topic visualisation techniques and information visualisation systems. Subjects could find relevant papers by examining them in the following areas: Title, Abstract, Keywords, and Content. Highly relevant papers could be simply detected from their title. However, where relevancy was difficult to determine then the abstract, keywords or content were explored.

Once they found a relevant paper, they were instructed to save the abstract of the paper to a local directory on their PC. After Task 1 was completed, subjects were asked to complete a brief spatial memory test. For this memory test, subjects were asked to sketch the shape of the visualised semantic space as best they could from their memory. The test was designed to find out what subjects could remember after having searched through the designed user interface.

For Task 2, subjects were asked to find five papers on the topic information structure, including topic structures, spatial structures and implicit structures. Once they found a relevant paper, they were instructed to save the abstract of the paper to a local directory on their PC. After Task 2 was completed, subjects were asked to name the cluster of papers in the visualised semantic space. The test was designed to find out whether subjects could summarise groups of papers associated with distinctive structure patterns and what naming schemes they might use. The meaningful finding could be used in future design.

- $\quad$ Post-test. A standard post-test questionnaire of Microsoft was used to assess usability issues and user satisfaction. The questionnaire consisted of three parts of questions about overall satisfaction, usability issues and user interface design.

\subsection{Results}

Qualitative and quantitative performance measures were collected during the test session. Correlation was computed between task performances and subjects' memory scores. In addition, usability and user satisfaction rating were assessed.

\subsubsection{Task Performance}

The associate memory and visual memory scores were assessed. Each memory test contained two repeated parts. Scores from the two parts were averaged and used. A one-way Analysis of Variance (ANOVA) was computed between memory scores and task performances. The task performance statistics and their correlation with memory scores are presented in table 1 . The number of abstracts saved by each individual was positively correlated with associative memory test in Task 1 . The table shows that associative memory was high significant $(\mathrm{P}<.001)$ in task 1 . However, visual memory was not significant in performing task 1 . The number of abstracts saved by each individual was negatively correlated with both memory tests in Task 2.

\begin{tabular}{|l|l|l|}
\hline Tasks & $\begin{array}{l}\text { Associative } \\
\text { Memory }\end{array}$ & $\begin{array}{l}\text { Visual } \\
\text { Memory }\end{array}$ \\
\hline Task 1 & $\begin{array}{l}\mathrm{r}=.85552 \\
\mathrm{p}=.003\end{array}$ & $\begin{array}{l}\mathrm{r}=.1796 \\
\mathrm{p}=.335\end{array}$ \\
\hline Task 2 & $\mathrm{r}=-.5750$ & $\mathrm{r}=-.6491$ \\
& $\mathrm{p}=.068$ & $\mathrm{p}=.041$ \\
\hline
\end{tabular}

Table 1: Task Performance Statistics and Their Correlation with Memory Score

\subsubsection{Navigation Strategies}

Many subjects directed their initial focus towards the central circle of the interface. Subjects would adjust the model to an easeful position to start navigation. Some subjects spent times to manipulate the virtual world before start to search. However, some subjects went straight to look for the paper without changing the position of the model. Branches with more nodes were normally examined in an initial stage. Branching points, where two branches were connected, were vital in their initial analyses. 


\subsubsection{Spatial Memory}

All subjects remembered the shape of the central circle. However, they remembered details vary from one another. Figure 4 shows the sketches of the structures from four different subjects.

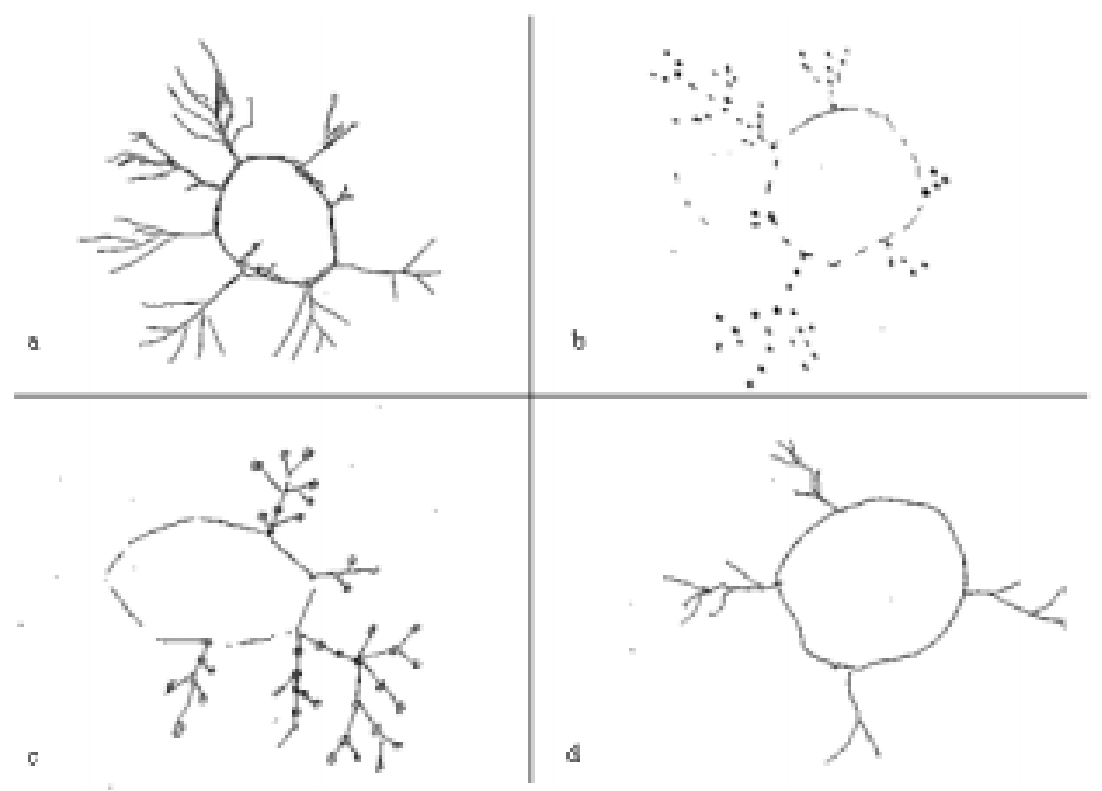

Figure 4: Subject's Sketches of the Semantic Information Space Searched during the Study

In (a), the subject was able to remember most details about the branches surrounding and strokes inside the central circle. The details were also the most accurate. The subject who sketched this structure achieved the highest score in task one. In (b), the subject drew less detail structure. However, the subjects emphasised each node in his drawing. He gave more detail in the branches that he had been visited for sometimes. In (c), although the sketch was not accurate, the subject sketched the branches that he had been focused and searched on. He drew in more detail at the first branch he started and spent times for it. In (d), the structure was less in detail and accuracy. The subject achieved the lowest score in Task 1.

\subsubsection{Categorisation and Abstraction}

The categorisation and abstraction task helps us understand how individuals would refer to distinctive structural patterns and what features associated with these patterns are useful in future design. All subjects were able to give names to structural patterns. Some subjects wanted to check at each cluster again before they could provide a name. Some subjects gave the cluster names from things that they are familiar and easy to remember such as comet, dolphin head and western. However, some subjects used a content-based naming scheme. For example, the names such as "visualisation", "user interface", and "interaction technique" were given to clusters that contain this information. The central circle is named as general, "mothership's orbit", "universe", and "main ring".

\subsubsection{User Satisfaction}

The post-test questionnaire was used to assess usability issues and user satisfaction. The questionnaire consists of three parts of questions about overall satisfaction, usability issues and user interface design. The details of the validated questionnaire are described in [8].

The majority of the subjects would recommend this system to others, on average (Mean 3.30, S.D. = 1.49). The scores were slightly below the average on the question of like the system or use it on a regular basis (see Table 2). 


\begin{tabular}{|l|l|l|}
\hline $\begin{array}{l}\text { Global Appeal (all ratings are on a 1-5 Likert scale, with 1=negative } \\
\text { and 5 = positive) }\end{array}$ & Mean & S.D. \\
\hline I would recommend this software to others. & 3.30 & 1.49 \\
\hline I like it. & 2.90 & 1.20 \\
\hline I would use this software on a regular basis & 2.78 & 1.56 \\
\hline
\end{tabular}

Table 2: Global Appeal Rating for the User Interface

Most of the subjects described the user interface as "original", "intuitive", "imaginative" and "fun". Some found it lacked predictability. Few found it was confusing and boring (see Table 3).

\begin{tabular}{|l|l|l|}
\hline Design Satisfaction & Mean & S.D. \\
\hline Original & 4.00 & 1.07 \\
\hline Intuitive & 3.50 & 1.35 \\
\hline Imaginative & 3.40 & 0.84 \\
\hline Fun & 3.20 & 1.55 \\
\hline Predicable & 2.80 & 1.55 \\
\hline Confusing & 2.20 & 1.62 \\
\hline Not my type of program & 2.00 & 1.83 \\
\hline Boring & 1.40 & 1.17 \\
\hline
\end{tabular}

Table 3: Design Satisfaction Ratings for the User Interface

Many subjects said that the purpose of the software was clear. However, the other three usability scores were slightly below the average (see Table 4).

\begin{tabular}{|l|c|c|}
\hline Usability & Mean & S.D. \\
\hline Right when I start, the purpose of the software was clear. & 3.80 & 1.03 \\
\hline Right when I started, I knew what I could do. & 2.90 & 1.29 \\
\hline It was easy to get where I wanted to go. & 2.80 & 1.03 \\
\hline Each area of the software was clearly marked to indicate my location. & 2.20 & 0.79 \\
\hline
\end{tabular}

Table 4: Usability Satisfaction Ratings for the User Interface

Online appeal factor ratings were above average. Many subjects liked the unique interface design (Mean $=4.20$, S.D. $=0.63)$. Average rating was low in familiarity.

\begin{tabular}{|l|c|c|}
\hline Online Appeal & Mean & S.D. \\
\hline This software feels unique (or different). & 4.20 & 0.63 \\
\hline This software provides a shared experience (or community). & 4.00 & 0.89 \\
\hline This software is timely (or up-to-date). & 3.89 & 1.05 \\
\hline This software is responsive (not too slow). & 3.50 & 1.18 \\
\hline This software is mentally challenging. & 3.44 & 1.01 \\
\hline This software is personalised/customisation. & 3.38 & 1.69 \\
\hline This software has appealing graphics. & 3.33 & 1.22 \\
\hline This software provides a detailed environment to interact with & 3.30 & 1.49 \\
\hline
\end{tabular}


An Empirical Study of Memory and Information Retrieval with a Spatial User Interface

\begin{tabular}{|l|c|c|}
\hline Online Appeal & Mean & S.D. \\
\hline This software provides valuable information. & 3.25 & 1.39 \\
\hline This software is easy to use. & 3.20 & 1.14 \\
\hline This software uses cutting edge technology. & 3.17 & 0.98 \\
\hline This software feels familiar. & 2.20 & 1.75 \\
\hline
\end{tabular}

Table 5: Satisfaction Rating for the Online Appeal of the User Interface

\section{Discussion}

Our statistical results show a strong positive correlation between memory scores and Task 1 performance as predicted. However, a negative correlation was found with consistency between visual memory scores and Task 2 performance. This may have been due to Task 2 being more difficult in finding a particular topic, especially where the test subject was unfamiliar with that topic. This resulted in the observation that subjects needed to explore a paper's content more fully. Further research testing could be done on subjects who have a specialist background that matches the content. New user models and workload assessment tools such as NASA TLX [11], which assesses Mental Demands, Physical Demands, Temporal Demands, Performance, Effort, and Frustration, will be examined in future experiments.

From our observation of navigation strategy, it seemed that people, who understood how the structure was semantically organised, developed good navigation strategies. This resulted in faster and an increased number of successful searches.

The sketches of the overall structure provide an indication of how subjects seemed to develop navigation strategies. Those who produced more detailed drawings appear to perform better tasks. The subject's memory may be influenced not only by information visualisation but also by differences in individual ability and strategy.

When abstracting information from the overall visualised semantic model, people gave it descriptive names according to what it reminded them of. We believe that people used these names as their personal landmarks.

The user satisfaction, usability issues and user interface design ratings were above the average. When people performed the task successfully they would be satisfied with the design and the usability rating would be high. This simple but important user satisfaction factor affects the likelihood of interface usage. It was suggested by some subjects that the interface should show where they were or where they had been in order to help them navigate easier. This possibility will be explored more fully in on-going research.

\section{Conclusion}

Virtual environments present a rich field of methodological opportunities for research on human cognition, specifically spatial ability. Our purpose in this study was to investigate the factor of individuals' abilities in information seeking in virtual environments. We examined the findings of other researchers in human factors influencing the design of the user interface for a large information space. We focused on the process of how memory acquires and retrieves visual information. Within this framework we undertook memory tests and assessed them against task performance in the virtual environment.

Our findings indicate that while associative memory was strongly correlated with the first task, visual memory was negatively correlated with the second task. Both correlations were statistically significant. This verifies that a key design element of an effective and usable 3D interface relies on human memory factors being taken into account which tends to equalise users of differing abilities.

\section{Acknowledgement}

This work was in part funded by the Brunel Research Initiative \& Enterprise Fund and by EPSRC Multimedia and Networking Applications Programme (GR/L61088).

\section{References}

1. Chen C. Bridging the gap: The use of pathfinder networks in visual navigation. Journal of Visual Language and Computing 1998, pp 267-286

2. Chen C. Structuring and visualising the WWW with Generalised Similarity Analysis. In Proceedings of Hypertext, New York: ACM Press, 1997, pp 177- 186 
3. Chen C. and Czerwinski M. Spatial ability and visual navigation: an empirical study. The New Review of Hypermedia and Multimedia, 1997, pp 67-90

4. Chun M.M. and Jiang Y. Contextual Cueing: Implicit Learning and Memory of Visual Context Guides Spatial Attention. Cognitive Psychology 36, Academic Press, 1998, pp 28-71

5. Darken R.P. and Sibert J.L. A toolset for navigation in virtual environments. In: User Interface Software and Technology, Atlanta, GA: ACM Press, 1993, pp 157-165

6. Darken R.P. and Sibert J.L. Wayfinding strategies and behaviors in large virtual worlds. In: Proceedings of CHI'96, ACM 1996, pp 142-149

7. Eckstrom R.B., French J.W., Harman H.H. and Derman D. Kit of factor-referenced cognitive test. Princeton, NJ: Educational Testing Service, 1976

8. Kanerva A., Keeker K., Risden K., Schuh E. and Czerwinski M. Web usability research at Microsoft Corporation. In: J. Ratner, E.Grosse and C. Forsythe (eds.), Human Factors for World Wide Web Development, New York: Lawrence Erlbaum, 1997, pp 207-224

9. Leplow B., Höll D., Zeng L. and Mehdorn M. Spatial Orientation and Spatial Memory Within a 'Locomotor Maze' for Humans. In C.Freksa, C.Habel and K.F. Wender (Eds.): Spatial Cognition, An Interdisciplinary Approach to Representing and Processing Spatial Knowledge. LNAI 1404, SpringerVerlag, 1998, pp 429-446

10. Mallot H.A., Gillner S., van Veen H.A.H.C. and Bülthoff H.H. Behavioural experiments in spatial cognition using virtual reality. In C.Freksa, C.Habel and K.F. Wender (Eds.): Spatial Cognition, An Interdisciplinary Approach to Representing and Processing Spatial Knowledge. LNAI 1404, SpringerVerlag, 1998, pp 447-467

11. NASA Ames Research Center, Human Performance Research Group. NASA Task Load Index (TLX). WWW: http://cseriac.flight.wpafb.af.mil, 1999

12. Swan R.C. and Allan J. Aspect Windows, 3-D Visualizations, and indirect comparisons of information retrieval systems. In: Proceedings of the $21^{\text {st }}$ annual international ACM SIGIR conference on Research and development in information retrieval, 1998, pp 173-181 